\title{
Topologically protected defect states in open photonic systems with non-hermitian charge-conjugation and parity-time symmetry
}

\author{
Simon Malzard, ${ }^{1}$ Charles Poli, ${ }^{1}$ and Henning Schomerus ${ }^{1,2}$ \\ ${ }^{1}$ Department of Physics, Lancaster University, Lancaster, LA1 4YB, United Kingdom \\ ${ }^{2}$ Max-Planck-Institut für Physik komplexer Systeme, 01187 Dresden, Germany
}

(Dated: November 11, 2015)

\begin{abstract}
We show that topologically protected defect states can exist in open (leaky or lossy) systems even when these systems are topologically trivial in the closed limit. The states appear from within the continuum, thus in absence of a band gap, and are generated via exceptional points (a spectral transition that occurs in open wave and quantum systems with a generalized time-reversal symmetry), or via a degeneracy induced by charge-conjugation-symmetry (which is related to the pole transition of Majorana zero modes). We demonstrate these findings for a leaking passive coupled-resonator optical waveguide with asymmmetric internal scattering, where the required symmetries (non-hermitian versions of time-reversal symmetry, chirality and charge-conjugation) emerge dynamically.
\end{abstract}

PACS numbers: 03.65.Vf, 42.60.Da, 42.55.Sa, 73.20.At

Fundamental symmetries appear in a new light when they are discussed within the context of open systems, where particles escape via leakage to the outside world or are absorbed within the material. In these situations one typically encounters decaying normal modes that can be described via the complex eigenfrequencies $\omega_{n}$ of an effective non-hermitian Hamiltonian, with $H \neq H^{\dagger}$. This description applies, e.g., on the level of the Helmholtz equation for dielectric microresonators or photonic crystals, where leaky losses enter through the boundary conditions while absorption renders the refractive index complex [1]. Such systems still obey reciprocity, $H=H^{T}$, while the antiunitary time-reversal symmetry $\mathcal{T} H \mathcal{T}=H^{*} \neq H^{T}=H$ is in general broken.

There has been much recent interest in open settings where a generalized antiunitary symmetry $\mathcal{P} \mathcal{T} H \mathcal{P} \mathcal{T}=H$ still exists $[2-6]$. Here $\mathcal{P}$ stands for parity, in this context understood to be a unitary involution with $\mathcal{P}^{2}=1$ that is often realized by a geometric reflection or inversion. True $\mathcal{P} \mathcal{T}$ symmetry requires amplifying (active) regions, arranged such that they are mapped via $\mathcal{P}$ onto corresponding absorptive parts, while leakage needs to be negligible. Eigenfrequencies $\omega_{n}$ then are either real or occur in a complex-conjugated pair [7], giving rise to a novel type of mode competition in lasers [8-12]. The more general case of a symmetry

$$
\mathcal{P} \mathcal{T}(H+i \gamma) \mathcal{P} \mathcal{T}=H+i \gamma
$$

with a finite offset $\gamma$ also encompasses suitably arranged passive systems where $\mathcal{P}$ now transforms between regions of different losses, while uniform leakage is also admitted. The constraints on the spectrum then apply to the shifted frequencies $\Omega_{n}=\omega_{n}+i \gamma$, which are either real or appear along with a partner $\Omega_{n}^{*}$. Among the many applications, these features can be used, e.g., to engineer band structures in periodic media where the dispersion is still effectively real, or posses some well-defined additional complex branches [13-16].
The advent of topological insulators and superconductors $[17,18]$ has taught us that the classification of universality classes in terms of time-reversal symmetry is incomplete. Two related symmetries, chirality [19, 20] and charge conjugation [21], need to be accounted for to identify band structures associated with finite topological quantum numbers [22], with the most prominent consequence being the formation of spatially localized defect states at interfaces (points, edges or surfaces) between topologically distinct domains. In particular, a unitary chiral anti-symmetry $\mathcal{X}\left(H-\Omega^{(0)}\right) \mathcal{X}=-\left(H-\Omega^{(0)}\right)$ enforces the spectrum to be symmetric around a central frequency $\Omega^{(0)}$, giving rise to frequency pairs $\Omega^{(0)} \pm \Omega_{n}$. A similar spectral constraint is also enforced by the antiunitary charge-conjugation symmetry $\mathcal{C}=\mathcal{T} \mathcal{X}$ that can appear in superconducting systems; this yields pairs $\Omega^{(0)}+\Omega_{n}, \Omega^{(0)}-\Omega_{n}^{*}$ and stabilizes unpaired resonances (broadened Majorana zero modes) at $\operatorname{Re} \Omega_{n}=0[26-$ 28]. Within this conventional classification, a necessary requirement for topological nontriviality is the existence of a band gap, into which the defect states then fall. The nascent field of topological photonics [29-44] has embarked to realize photonic analogues of these symmetries, while the (beneficial or detrimental?) role of non-hermitian loss and gain has only been considered in settings which are already topological in the hermitian limit [44-51].

Here we identify, for the simple example of a coupledresonator chain, a mechanism by which topologically protected defect states can appear in open (non-hermitian) systems even when their closed (hermitian) limit is topologically trivial. The defect states form at an interface of two regions with different non-hermiticity, and appear from the continuum of the band structure via two distinct symmetry-protected spectral transitionswhere one is associated with $\mathcal{P} \mathcal{T}$-symmetry (thus, nonhermitian time-reversal symmetry), while the other is associated with $\mathcal{P C}$-symmetry (thus, the analogously gen- 
(a)

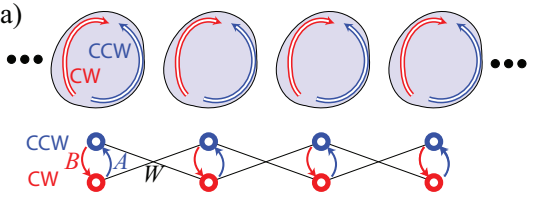

(b)

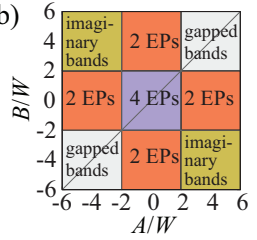

(c)
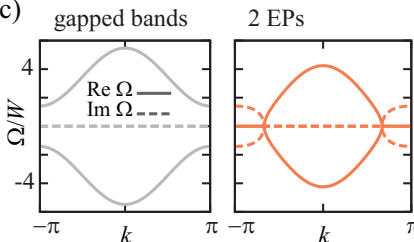

(d)
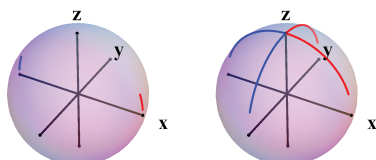

4 EPs

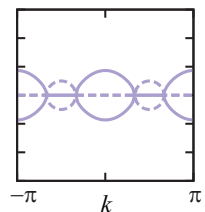

imaginary bands
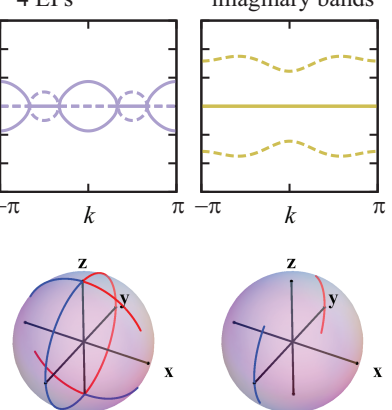

FIG. 1. (color online) (a) Coupled-resonator waveguide with internal asymmetric scattering (couplings $A$ and $B$ ) between a counterclockwise $(\mathrm{CCW})$ and a clockwise $(\mathrm{CW})$ wave component, and coupling $W$ between CCW and CW components in neighboring resonators. For real couplings this system realizes non-hermitian versions of time-reversal symmetry, chirality and charge conjugation. (b) Phase diagram for the bulk dispersion (6). The dispersion can be real and gapped, exhibit 2 or 4 exceptional points (EPs) at which real and imaginary branches meet, or be fully imaginary. In the examples in (c), $A / W=4$ and $B / W=3$ (real gapped dispersion), $A / W=4$ and $B / W=1$ (2EPs), $A / W=1$ and $B / W=-1$ (4EPs), as well as $A / W=4$ and $B / W=-3$ (imaginary dispersion). (d) Corresponding representation of the complex bands on the Bloch sphere (upper band $\Omega_{+}$: red, lower band $\Omega_{-}$: blue).

eralized charge-conjugation symmetry that arises from the simultaneous presence of a chiral symmetry). Thus, robust states as desired for topological photonics can be obtained by combining symmetries with non-hermitian effects that go beyond the universal classification of electronic systems.

Dynamical realization of non-hermitian parity-time and charge-conjugation symmetry. - We first describe how the required symmetries can be implemented in a simple passive resonator chain, with losses solely provided due to leakage but without any need of absorption or amplification (which then also translates to analogous open quantum systems). This can be achieved in a coupled-resonator optical waveguide (CROW) [5254] which consists of identical asymmetric cavities [55], as sketched in Fig. 1(a). Each individual resonator features two modes - a counterclockwise (CCW) propagating mode with amplitude $a_{n}$ and a clockwise (CW) propagating mode with amplitude $b_{n}$, which we group into a vector $\psi_{n}=\left(a_{n}, b_{n}\right)^{T}$. Resonator arrays in which these two modes are well decoupled feature in setups that realize photonic topological edge states in analogy to the quantum-Hall effect [34, 38]. In our setting, however, the

internal coupling between these modes is desired, and the key feature is that this coupling can be made asymmetric by opening the system, even if no magnetic field is applied - as has been established in recent works on individual resonators [55-60]. The coupling of the modes is then described by a nonhermitian internal Hamiltonian $[55,60]$

$$
h=\left(\begin{array}{cc}
\Omega^{(0)} & A \\
B & \Omega^{(0)}
\end{array}\right),
$$

where the constants $A \neq B^{*}$ and $\Omega^{(0)}$ account for the asymmetric internal scattering and the losses within the cavity. Throughout the chain, the coupling between adjacent resonators is dominantly between $\mathrm{CCW}$ and $\mathrm{CW}$ waves, so that the coupling matrix is $[60,61]$

$$
t=\left(\begin{array}{cc}
0 & W \\
W & 0
\end{array}\right)
$$

In coupled-mode approximation, the stationary wave equation then takes the form

$$
\omega \psi_{n}=h \psi_{n}+t\left(\psi_{n+1}+\psi_{n-1}\right)
$$

which admits Bloch solutions $\psi_{n}=\exp (i k n) \Psi$. The associated Bloch Hamiltonian is

$$
h(k)=\left(\begin{array}{cc}
\Omega^{(0)} & A+2 W \cos (k) \\
B+2 W \cos (k) & \Omega^{(0)}
\end{array}\right),
$$

and leads to the dispersion relation $\omega_{ \pm}(k)=\Omega^{(0)}+$ $\Omega_{ \pm}(k)$,

$$
\Omega_{ \pm}(k)= \pm \sqrt{(A+2 W \cos k)(B+2 W \cos k)},
$$

where the subscript \pm labels two bands. The symmetry about $\Omega^{(0)}$ is a consequence of a chiral symmetry with $\mathcal{X} \psi_{n}=\sigma_{z} \psi_{n}$, which maps the bands onto each other. The chiral symmetry is thus realized by the freedom of the relative sign of the CCW and CW amplitudes (a gauge freedom compatible with time-reversal symmetry which generically appears in systems with two mutually coupled sublattices).

As shown by exact numerical calculations [60, 61], for representative resonator geometries $A, B$ and $W$ are almost real, and can be further tuned towards real values by adjustments of a few shape parameters. We thus neglect the imaginary parts of these parameters. Apart from an offset $\gamma=i \operatorname{Im} \Omega^{(0)}$, the dispersion is then either real (in some range of $k$ ) or purely imaginary (in the complementary range of $k$ ). These $k$ ranges are joined by degeneracies, known as exceptional points [62-66], where $\omega_{ \pm}(k)=\Omega^{(0)}$, thus $\cos k=-A / 2 W$ or $\cos k=-B / 2 W$. A completely real dispersion with a gap is achieved if $|A / 2 W|>1$ and $|B / 2 W|>1$, provided $A B>0$ [see the phase diagram in Fig. 1(b) and representative dispersions in Fig. 1(c)]. 
The underlying symmetry can be made explicit by a basis change, $\phi_{n}=2^{-1 / 2}\left(i \sigma_{x}+\mathbb{1}\right) \psi_{n}$ where $\sigma_{x}$ is a Pauli matrix, after which the Bloch Hamiltonian takes the form

$$
\tilde{h}(k)=\frac{1}{2}\left(\begin{array}{cc}
2 \Omega^{(0)}+i(A-B) & A+B+4 W \cos k \\
A+B+4 W \cos k & 2 \Omega^{(0)}-i(A-B)
\end{array}\right) .
$$

The Hamiltonian is now symmetric, as required by reciprocity (which is hidden in the $\mathrm{CCW} / \mathrm{CW}$ basis since propagating waves are complex), and furthermore exhibits a passive $\mathcal{P} \mathcal{T}$ symmetry (1) with $\mathcal{P}=\sigma_{x}$, and $\gamma=-\operatorname{Im} \Omega^{(0)}$. The chiral symmetry is transformed to $\mathcal{X}=\sigma_{y}$, and commutes with $\mathcal{P} \mathcal{T}$. This realizes all the symmetries mentioned in the introduction, including $\mathcal{P C}=\mathcal{P} \mathcal{T} \mathcal{X}$, with respect to the central frequency $\Omega^{(0)}$. From here on, we work in terms of the shifted frequencies $\Omega=\omega-\Omega^{(0)}$, for which the dispersion is directly given by Eq. (6).

Having established these symmetries we now return to the $\mathrm{CCW} / \mathrm{CW}$ basis and discuss topological aspects of the band structure. For this we consider the $k$ dependence of the Bloch vectors $\Psi(k)$, which we interpret as pseudospins with polarization vector $\vec{P}=\left\langle\left(\sigma_{x}, \sigma_{y}, \sigma_{z}\right)\right\rangle$. In the hermitian limit $B=A$ (both real), the two bands $\Omega_{ \pm}(k)= \pm(A+2 W \cos k)$ arise from $k$-independent pseudospins $\Psi_{ \pm}=2^{-1 / 2}(1, \pm 1)^{T}$, with $\vec{P}=( \pm 1,0,0)$ pointing along the $x$ axis. The absence of any winding of the pseudospin renders the system topologically trivial, so that we do not expect any defect states in the presence of interfaces, even if there is a gap. In the non-hermitian case, we can write $\Psi_{ \pm}(k) \propto\left(A+2 W \cos k, \Omega_{ \pm}(k)\right)^{T}$. As shown in Fig. 1(d), the polarization vector now acquires $k$ dependence; it is confined to the $x z$ plane when the dispersion is real and to the $y z$ plane when the dispersion is imaginary. These branches are again joined at the exceptional points, where $\vec{P}$ points up or down along the $z$ axis, with $\vec{P}_{-}(k)=R_{z}(\pi) \vec{P}_{+}(k)$ related by a $\pi$ rotation about the $z$ axis. In particular, the way these points are connected depends on whether $A>B$ or $A<B$ (with the two cases related by a rotation $R_{x}(\pi)$ by $\pi$ about the $x$ axis). Does the system now admit defect states?

Defect states. - In order to answer this question, we create a defect in the chain by inverting the orientation of the resonators in half of the system [see Fig. 2(a)]. From the traditional perspective of hermitian systems, the defect cannot be classified as topological, and does not give rise to any defect states. In the non-hermitian setting, we will see that the defect acquires topological features in a spectral phase transition at which localized defect states emerge [as illustrated in Fig. 2(b)]. The phase transition takes the form of a $\mathcal{P} \mathcal{T}$-induced exceptional point along one part of the phase boundary, while it is associated with a $\mathcal{P C}$-induced degeneracy along the other parts of the phase boundary [this is summarized in Figs. 2(c,d), to which we refer throughout the remaining discussion].

It is easy enough to identify the conditions for the for- (a)

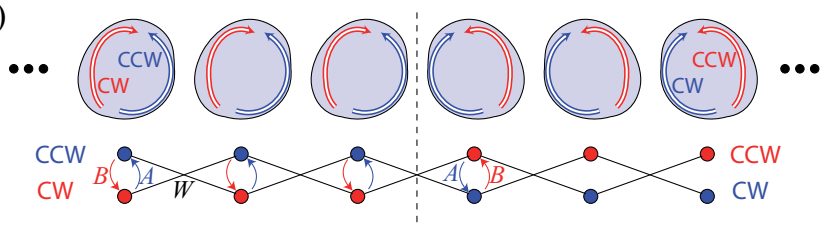

(b)
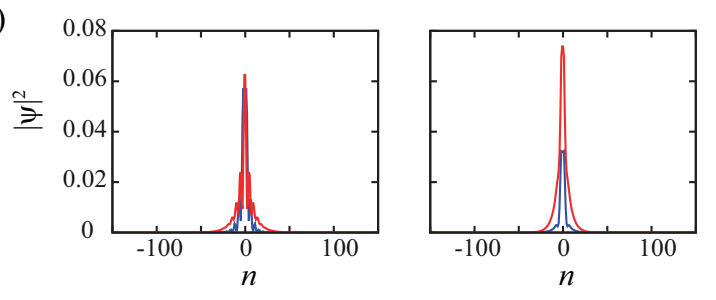

(c)

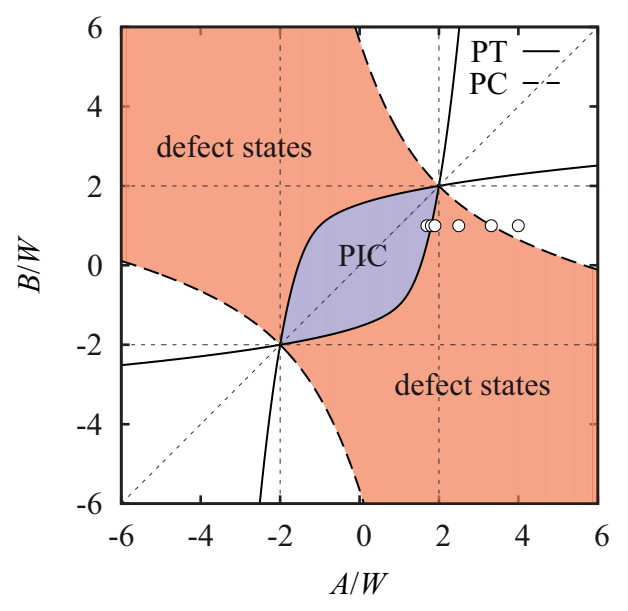

(d)
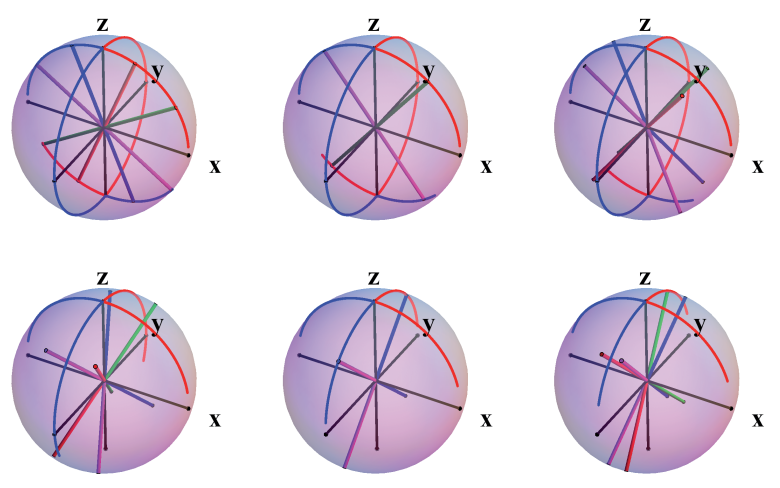

FIG. 2. (color online) (a) Coupled-resonator waveguide with a defect, created by inverting the orientation of the resonators in half of the system. In the closed limit, the system is trivial, and the defect does not create any bound states. (b) Defect states in a system of 300 resonators, with $A / W=1.9$ (left panel) and $A / W=2.5$ (right panel), while $B / W=1$. (c) Phase diagram indicating the existence of defect states, as well as their extended-state precursors (realizing perfect interband transitions, PIC). The boundaries of the defect phase are given by degeneracy conditions. At the PT boundary, the extended PIC states bifurcate into pairs $\Omega_{n}, \Omega_{n}^{*}$ of defect states that are related by the $\mathcal{P} \mathcal{T}$ symmetry. At the PC boundary one encounters a degeneracy of charge-conjugated partner states $\Omega_{n},-\Omega_{n}^{*}$, beyond which the defect states are non-normalizable. (d) Bloch-sphere position of the defect states (rods) relative to the bulk dispersion (lines) [parameters $A / W=1.7,1.81$ (PT), 1.9, 2.5, 3.32 (PC), 4 with $B / W=1$, as indicated by the white circles in (c)]. 
mation of defect states. In presence of the defect, the wave equation takes the form

$$
\Omega \psi_{n}=h_{n} \psi_{n}+t\left(\psi_{n+1}+\psi_{n-1}\right),
$$

where now $h_{n}=\left(h-\Omega^{(0)}\right)$ for $n<0$ (left half of the chain) and $h_{n}=\left(h-\Omega^{(0)}\right)^{T}$ for $n \geq 0$ (right half of the chain). At any fixed $\Omega$, in each half of the system the solutions are still obtained from a superposition of Bloch waves with

$$
2 W \cos k_{ \pm}=c_{ \pm}=-\frac{A+B}{2} \pm \sqrt{\frac{(A-B)^{2}}{4}+\Omega^{2}},
$$

associated with pseudospins

$$
\Psi_{ \pm}^{(L)}(\Omega) \propto\left(\begin{array}{c}
A+c_{ \pm} \\
\Omega
\end{array}\right), \quad \Psi_{ \pm}^{(R)}(\Omega) \propto\left(\begin{array}{c}
\Omega \\
A+c_{ \pm}
\end{array}\right)
$$

Each of the values $c_{+}, c_{-}$are associated with a pair of Bloch waves with propagation factors $\exp \left(i k_{ \pm}\right)=\lambda_{ \pm}$, $\exp \left(-i k_{ \pm}\right)=\left(\lambda_{ \pm}\right)^{-1}$, where we choose $k_{ \pm}$such that $\left|\lambda_{ \pm}\right| \geq 1$ if $k_{ \pm}$is complex. We now match the solutions with propagation factor $\exp \left(i k_{ \pm}\right)$in the left part to the solutions with propagation factor $\exp \left(-i k_{ \pm}\right)$in the right part. One then finds the condition

$$
(\Omega-2 W)^{2} \Omega-A B \Omega=(A-B)^{2} W / 2
$$

for defect states with a symmetric wavefunction, and

$$
-(\Omega+2 W)^{2} \Omega+A B \Omega=(A-B)^{2} W / 2
$$

for defect states with an antisymmetric wavefunction. Due to the $\mathcal{P} \mathcal{T}$ symmetry, solutions are again either real or appear in complex conjugated pairs. Furthermore, chirality maps $\Omega \rightarrow-\Omega$, corresponding to a transformation between symmetric and antisymmetric wavefunctions. Thus, the defect states come in a quadruple of frequencies $\Omega_{n}, \Omega_{n}^{*},-\Omega_{n},-\Omega_{n}^{*}$. The condition that the corresponding wave function indeed decays leads to the phase diagram in Fig. 2(c).

In this phase diagram, the hermitian case $A=B$ defines a diagonal. The defect states are confined to a region away from the diagonal, which is bounded by two different transitions. Along the curves labeled PT, where $27(A+B)^{4}=16 A^{2} B^{2}\left(1+A B / W^{2}\right)+8\left(8 W^{2}+\right.$ $9 A B)(A+B)^{2}$, a pair of real solutions $\Omega_{n}$ of Eq. (11) bifurcates into a pair of complex-conjugated solutions. Before this exceptional point, the solutions are real, with $\left|\lambda_{ \pm}\right|=1$, and describe the scattering of an incoming extended state in one band into an outgoing extended state in the other band. This region of perfect interband conversion is labeled PIC. At the exceptional point, the propagation factors $\lambda_{+}$of the two solutions coalesce, and so do the factors $\lambda_{-}$; beyond the exceptional point we then have $\left|\lambda_{ \pm}\right|>1$, giving rise to properly normalizable defect states. The same scenario occurs simultaneously for the chirality-related solutions of Eq. (12). The second kind of transition appears along the curves labeled PC, where $A^{2}+6 A B+B^{2}=32 W^{2}$. There, a complex solution $\Omega_{n}$ of Eq. (11) coalesces with a charge-conjugated solution $-\Omega_{n}^{*}$ of Eq. (12), meaning that they are purely imaginary. This is similar to the pole transition of broadened Majorana zero-modes, which are then pinned to the imaginary axis and become their own charge-conjugated partner. These transitions also occur in skew-Hamiltonian ensembles governing the topological transitions in Josephson junctions [67]. In the present problem, the PC transition signals the point where the matching conditions can only be fulfilled by combining decaying with increasing wavefunctions, which occurs when one of the wave numbers $k_{+}, k_{-}$crosses the real axis.

At both types of transition, the defect states therefore interact with the real branch of the dispersion relation $\Omega_{ \pm}(k)$ (for the exceptional points along the $\mathrm{PT}$ boundary), or with the purely imaginary branch of this dispersion relation (for the charge-conjugation-induced degeneracy along the PC boundary). On the level of the wavefunctions, this interaction is again revealed via the corresponding polarization vectors. Focussing on the wave function in the left part of the system, we have

$$
\vec{P}_{-}^{(L)}(\Omega)=-\vec{P}_{+}^{(L)}\left(\Omega^{*}\right)
$$

for the two partial waves $\Psi_{ \pm}^{(L)}$ given in Eq. (10), while the $\mathcal{P} \mathcal{T}$ and chiral symmetries relate

$$
\begin{aligned}
\vec{P}_{ \pm}^{(L)}\left(\Omega^{*}\right) & =-R_{y}(\pi) \vec{P}_{ \pm}^{(L)}(\Omega), \\
\vec{P}_{ \pm}^{(L)}(-\Omega) & =R_{z}(\pi) \vec{P}_{ \pm}^{(L)}(\Omega),
\end{aligned}
$$

where $R_{a}$ denotes a $\pi$ rotation about axis $a$. [We also have $\vec{P}_{ \pm}^{(R)}(\Omega)=R_{x}(\pi) \vec{P}_{ \pm}^{(L)}(\Omega)$.] In Fig. 2(d), we show how these polarization vectors interact. In the PIC phase, each state corresponds to a pair of opposite vectors $\vec{P}_{ \pm}^{(L)}$ confined to the $x z$ plane (the locus of the real dispersion branch), with chirality-related partner states connected by a $\pi$ rotation about the $z$ axis. At the PT transition, the vectors bifurcate and move out of the $x z$ plane. In the defect phase, the two vectors $\vec{P}_{ \pm}^{(L)}$ for a given defect state make an angle, but remain related by a $\pi$ rotation about the $y$ axis. The partner state with frequency $\Omega^{*}$ points into the opposite direction, while the chirality-related states are still obtained by a $\pi$ rotation about the $z$ axis. At the PC transition, each state collides with a charge-conjugated partner at a point in the $y z$ plane (the locus of the imaginary dispersion branch). These interactions all occur at symmetry-protected positions, which renders the defect phase topologically stable. For numerical verification of this robustness in finite and disordered systems see [68].

In summary, robust defect states can exist in open systems that are topologically trivial in the closed limit. We illustrated this for a leaky optical resonator chain 
where defect states appear at an interface between regions in which hermiticity is broken in different ways (in contrast, non-hermiticity is not sufficient to create edge states at the end of a finite sample). The states are topologically protected as they arise in spectral phase transitions that are linked to the spontaneous breaking of fundamental symmetries (parity-time symmetry and charge-conjugation symmetry) for sufficient degree of non-hermiticity in the system. The required symmetries are realized when the couplings in the propagating wave basis are real. As this does not require any absorption, the formation mechanisms described here also translate to analogous geometrically open quantum systems, including electronic systems which are suitably coupled to external reservoirs. Our observations raise new questions, such as whether it is possible to characterize these systems in terms of topological quantum numbers, and more generally whether they can be understood by a suitable extension of the conventional topological classification of closed systems.

We thank Jan Wiersig for useful discussions and comments. This research was supported by EPSRC via grant EP/J019585/1. The data created during this research is openly available [71].

[1] H. Cao and J. Wiersig, Rev. Mod. Phys. 87, 61 (2015).

[2] A. Guo, G. J. Salamo, D. Duchesne, R. Morandotti, M. Volatier-Ravat, V. Aimez, G. A. Siviloglou, and D. N. Christodoulides, Phys. Rev. Lett. 103, 093902 (2009).

[3] C. E. Rüter, K. G. Makris, R. El-Ganainy, D. N. Christodoulides, M. Segev, and D. Kip, Nat. Phys. 6, $192(2010)$.

[4] A. Regensburger, C. Bersch, M.-A. Miri, G. Onishchukov, D. N. Christodoulides, and U. Peschel, Nature (London) 488, 167 (2012).

[5] L. Feng, Y.-L. Xu, W. S. Fegadolli, M.-H. Lu, J. E. B. Oliveira, V. R. Almeida, Y.-F. Chen and A. Scherer, Nat. Mater. 12, 108 (2013).

[6] T. Eichelkraut, R. Heilmann, S. Weimann, S. Stützer, F. Dreisow, D. N. Christodoulides, S. Nolte and A. Szameit, Nat. Commun. 4, 2533 (2013).

[7] C. M. Bender, Rep. Prog. Phys. 70, 947 (2007).

[8] H. Schomerus, Phys. Rev. Lett. 104, 233601 (2010).

[9] S. Longhi, Phys. Rev. A 82, 031801(R) (2010).

[10] Y. D. Chong, L. Ge, and A. D. Stone, Phys. Rev. Lett. 106, 093902 (2011).

[11] L. Feng, Z. J. Wong, R.-M. Ma, Y. Wang, and X. Zhang, Science 346, 972 (2014).

[12] H. Hodaei, M.-A. Miri, M. Heinrich, D. N. Christodoulides, and M. Khajavikhan, Science 346, 975 (2014).

[13] K. G. Makris, R. El-Ganainy, D. N. Christodoulides, and Z. H. Musslimani, Phys. Rev. Lett. 100, 103904 (2008).

[14] S. Longhi, Phys. Rev. Lett. 103, 123601 (2009).

[15] H. Ramezani, T. Kottos, V. Kovanis, and D. N. Christodoulides, Phys. Rev. A 85, 013818 (2012).

[16] A. Szameit, M. C. Rechtsman, O. Bahat-Treidel, and M.
Segev, Phys. Rev. A 84, 021806(R) (2011).

[17] M. Z. Hasan and C. L. Kane, Rev. Mod. Phys. 82, 3045 (2010).

[18] X.-L. Qi and S.-C. Zhang, Rev. Mod. Phys. 83, 1057 (2011).

[19] J. J. M. Verbaarschot and I. Zahed, Phys. Rev. Lett. 70, 3852 (1993).

[20] J. J. M. Verbaarschot and T. Wettig, Ann. Rev. Nucl. Part. Sci. 50, 343 (2000).

[21] A. Altland and M. R. Zirnbauer, Phys. Rev. B 55, 1142 (1997).

[22] The full classification also includes spin-orbit coupling via variants with $\mathcal{T}^{2}=-1$ and considerations of dimensionality [23-25].

[23] S. Ryu, A. P. Schnyder, A. Furusaki, and A. W. W. Ludwig, New J. Phys. 12, 065010 (2010).

[24] J. C. Y. Teo and C. L. Kane, Phys. Rev. B 82, 115120 (2010).

[25] C. W. J. Beenakker, submitted to Rev. Mod. Phys. [arXiv:1407.2131].

[26] D. Pikulin and Y. Nazarov, JETP Letters 94, 693 (2012).

[27] D. I. Pikulin and Y. V. Nazarov, Phys. Rev. B 87, 235421 (2013).

[28] P. San-Jose, J. Cayao, E. Prada, and R. Aguado, arxiv:1409.7306 (2014).

[29] L. Lu, J.D. Joannopoulos, and M. Soljacic, Nat. Photonics 8, 821 (2014).

[30] S. Raghu and F. D. M. Haldane, Phys. Rev. A 78, 033834 (2008).

[31] Z. Wang, Y. D. Chong, J. D. Joannopoulos, and M. Soljacic, Phys. Rev. Lett. 100, 013905 (2008).

[32] Z. Wang, Y. Chong, J. D. Joannopoulos, and M. Soljacic, Nature (London) 461, 772 (2009).

[33] N. Malkova, I. Hromada, X. Wang, G. Bryant, and Z. Chen, Opt. Lett. 34, 1633 (2009).

[34] M. Hafezi, E. A. Demler, M. D. Lukin, and J. M. Taylor, Nat. Phys. 7, 907 (2011).

[35] K. Fang, Z. Yu, and S. Fan, Nat. Photonics 6, 782 (2012).

[36] T. Kitagawa, M. A. Broome, A. Fedrizzi, M. S. Rudner, E. Berg, I. Kassal, A. Aspuru-Guzik, E. Demler, and A. G. White, Nat. Commun. 3, 882 (2012).

[37] A. B. Khanikaev, S. H. Mousavi, W.-K. Tse, M. Kargarin, A. H. MacDonald, and G. Shvets, Nat. Mater. 12, 233 (2013).

[38] M. Hafezi, S. Mittal, J. Fan, A. Migdall, and J. M. Taylor, Nat. Photonics 7, 1001 (2013).

[39] M. C. Rechtsman, J. M. Zeuner, Y. Plotnik, Y. Lumer, D. Podolsky, F. Dreisow, S. Nolte, M. Segev, and A. Szameit, Nature (London) 496, 196 (2013).

[40] M. C. Rechtsman, J. M. Zeuner, A. Tünnermann, S. Nolte, M. Segev, and A. Szameit, Nat. Photonics 7, 153 (2013).

[41] R. Keil, J. M. Zeuner, F. Dreisow, M. Heinrich, A. Tünnermann, S. Nolte, and A. Szameit, Nat. Commun. 4, 1368 (2013).

[42] S. Mittal, J. Fan, S. Faez, A. Migdall, J. M. Taylor, and M. Hafezi Phys. Rev. Lett. 113, 087403 (2014).

[43] A. Poddubny,A. Miroshnichenko, A. Slobozhanyuk, and Y. Kivshar, ACS Photonics 1, 101 (2014).

[44] C. Poli, M. Bellec, U. Kuhl, F. Mortessagne, H. Schomerus, Nat. Commun. 6, 6710 (2015).

[45] M. S. Rudner and L. S. Levitov, Phys. Rev. Lett. 102, 065703 (2009)

[46] S. Diehl, E. Rico, M. A. Baranov, and P. Zoller, Nat. 
Phys. 7, 971 (2011).

[47] K. Esaki, M. Sato, K. Hasebe, and M. Kohmoto, Phys. Rev. B 84, 205128 (2011)

[48] Y. C. Hu and T. L. Hughes, Phys. Rev. B 84, 153101 (2011).

[49] H. Schomerus, and N. Yunger Halpern, Phys. Rev. Lett. 110, 013903 (2013)

[50] H. Schomerus, Optics Letters 38, 1912 (2013).

[51] J. M. Zeuner, M. C. Rechtsman, Y. Plotnik, Y. Lumer, S.Nolte, M. S. Rudner, M. Segev, and A. Szameit, Phys. Rev. Lett. 115, 040402 (2015).

[52] B. E. Little, S. T. Chu, J. Haus, H. A. Foresi, and J.-P. Laine, J. Lightwave Technol. 15, 998 (1997).

[53] N. Stefanou and A. Modinos, Phys. Rev. B 57, 12127 (1998).

[54] A. Yariv, Y. Xu, R. K. Lee, and A. Scherer, Opt. Lett. 24, 711 (1999).

[55] J. Wiersig, S. W. Kim, and M. Hentschel, Phys. Rev. A 78, 053809 (2008).

[56] J. Wiersig, A. Eberspächer, J.-B. Shim, J.-W. Ryu, S. Shinohara, M. Hentschel, and H. Schomerus, Phys. Rev. A 84, 023845 (2011).

[57] J. Wiersig, Phys. Rev. A 84, 063828 (2011).

[58] X. Yi, Y.-F. Xiao, Y.-C. Liu, B.-B. Li, Y.-L. Chen, Y.
Li, and Q. Gong, Phys. Rev. A 83, 023803 (2011).

[59] D. D. Scott and Y. N. Joglekar, Phys. Rev. A 85, 062105 (2012).

[60] J. Wiersig, Phys. Rev. A 89, 012119 (2014).

[61] H. Schomerus and J. Wiersig, Phys. Rev. A 90, 053819 (2014).

[62] T. Kato, Perturbation Theory for Linear Operators (Springer, New York, 1966).

[63] W. D. Heiss, Phys. Rev. E 61, 929 (2000).

[64] W. D. Heiss, J. Phys. A 45, 444016 (2012).

[65] M. V. Berry, Czech. J. Phys. 54, 1039 (2004).

[66] J. Wiersig, Phys. Rev. Lett. 112, 203901 (2014).

[67] C. W. J. Beenakker, J. M. Edge, J. P. Dahlhaus, D. I. Pikulin, S. Mi, and M. Wimmer, Phys. Rev. Lett. 111, 037001 (2013).

[68] For the numerical demonstration of robustness in finite and disordered systems see the Supplemental Material at URL, which includes Refs. [65, 66, 69, 70].

[69] H. Schomerus, K. M. Frahm, M. Patra, and C. W. J. Beenakker, Physica A 278, 469 (2000).

[70] H. Schomerus, Phys. Rev. A 79, 061801(R) (2009).

[71] The data is openly available at http://dx.doi.org/10.17635/lancaster/researchdata/23 